\title{
A SURVEILLANCE SYSTEM FOR ENHANCING THE SAFETY OF RESCUE TEAMS
}

The article summarizes preliminary results of the research and development of a system focused on enhancing the safety of teams participating in the integrated rescue system managing extraordinary events or crisis situations (fire, mass disaster, release of harmful industrial substances), and on the support in the course of training. Individual partial technical solutions are mentioned, which should lead to providing automatized telemetric monitoring equipment in a more resistant form making it possible to recognize the nature and intensity of the motion, including the determination of the topical and total energy outputs, monitoring of environmental parameters (temperature, smoke, etc.) and back analysis of the intervention course or training in real time, and the monitoring of health-physiological parameters and signalling risk conditions (physical exhaustion, stress, overheating, etc.) under extreme measures.

Keywords: Integrated rescue system, monitoring, surveillance system, safety.

\section{Introduction}

The contemporary world faces new challenges associated with the development of society, particularly the permanent and sustainable development, protection of the health and life of the population and environmental protection. The essential viewpoint includes the permanent flow of important data, their evaluation and final determination of appropriate decisions [1]. This doubtlessly also holds in the field of telemetry which includes a set of technologies and methods facilitating remote measurements of physical quantities including the transfer of the data measured. Bio-telemetric systems are also of importance from the operation stand point being based on the principles of monitoring the individual's psychophysiological condition applied to military disciplines and to monitoring workers in further heavyduty professions, such as operators of complex technical facilities (as, for example, nuclear power plants), professional chauffeurs of motor vehicles in long-distance transport and, last but not least, members of rescue teams [2 and 3].

During interventions by and training of rescue teams, the members are able to take advantage of wearable automatized monitoring equipment which could also be able to provide combined relevant data about the position, personal healthphysiological condition and environmental parameters in the surroundings of the monitored member of the rescue team even under extreme circumstances. These are very important parameters directly affecting the efficacy/quality of the intervention and safety of particular members of the team participating in the intervention.

The article dealing with the condition of the project studied within the framework of the safety research, with the aim to develop a functional sample of this bio-telemetric surveillance equipment for selected teams of the integrated rescue system, includes a brief outline of existing concepts of supporting systems for members of rescue teams throughout the world: those examples of systems were selected which exert their obvious application potential and thus, they are not a matter of academic projects only. The basic principal function of the surveillance equipment developed including partial results of the project is mentioned further.

\section{Surveillance supporting systems developed for rescue services}

LifeNet is a system aimed at the location of firefighters operating inside of complex buildings. It is based on a principle similar to that where the firefighters draw a guiding rope which, for example, serves to aid the rapid navigation through smoky environments where visual orientation is impossible, or can

\footnotetext{
* ${ }^{1}$ Zdenek Hon, ${ }^{2}$ Pavel Smrcka, ${ }^{2}$ Karel Hana, ${ }^{2}$ Jan Kaspar, ${ }^{2}$ Jan Muzik, ${ }^{2}$ Radek Fiala, ${ }^{2}$ Martin Viteznik, ${ }^{2}$ Tomas Vesely, ${ }^{2}$ Lukas Kucera,

${ }^{2}$ Tomas Kuttler, ${ }^{2}$ Radim Kliment, ${ }^{1}$ Vaclav Navratil

${ }^{1}$ Department of Health Care Disciplines and Population Protection, Faculty of Biomedical Engineering, Czech Technical University in Prague,

Czech Republic

${ }^{2}$ Joint Department of Biomedical Engineering Czech Technical University and Charles University in Prague, Faculty of Biomedical Engineering,

Czech Technical University in Prague, Czech Republic

E-mail: zdenek.hon@fbmi.cvut.cz
} 
also help find a member of the team attached to the second end of the same rope. The LifeNet system attempts to implement this concept under conditions using modern technologies. The firefighter wears equipment making it possible to hand throw, or automatized throwing, away of a few beacons (buoys) which subsequently serve as admission points. These beacons are able to detect particular members of the team in their vicinity with the use of an ultrasonic transmitter including their distance and position with respect to the beacon. Thanks to this the firemen can be located inside of extensive complexes. Miniaturized monitors can be connected to the equipment which are situated in the respirator of the firefighter and thanks to them, the firefighter performing the direct intervention can observe his position and the position of other members with respect to particular beacons. The firefighter location with the help of particular beacons is provided in cooperation with equipment attached to the firefighter's footwear. This equipment also includes a temperature sensor with a possibility of connecting to further sensors, as, e.g., an accelerometer, with the help of the I2C interface. Currently, this system is in the developmental prototype stage [4 and 5].

The MiTag (Medical information Tag) system is designed for acquiring data from a number of persons involved. However, its concept is related to the surveillance system for intervening teams. The system is based on the MiTag platform which comprises two wireless interfaces. The interface providing the communication with sensors forms the Body Area Network (BAN). The communication with the display is provided by a MESH type long-range network. The system also includes repeaters which can be thrown away along the path between the display unit and patient in the cases where direct attainability of the signal from the platform on the patient to the display is impossible. The protocol of the MESH network then redirects the flow of data through these repeaters, thus providing a theoretically unlimited distance, along which the data can be transferred. Many different sensors can be connected to the platform (GPS, pulsed oximetry, blood pressure or temperature sensors, ECG, etc.) [6].

The FireNet presents an architecture of a wireless network directly designed for needs of transferring data taken by sensors in the case of firefighting emergency operations. The Ad-Hoc type network is capable of its own reconfiguration as required of transferring data to the site established. Different types of sensors are connected to the network, situated on firefighters themselves or on some parts of their equipment, as for example, on vehicles. On vehicles there is also a GPS receiver which can be used for the location of firefighters, and with the help of the network itself, it is then possible to partially locate relative positions of particular points. Data acquired is transferred to the display equipment at the commander of the intervention on the one hand, and with the help of internet, to the firefighting central command on the other. Thereafter, both groups have admission to online data acquired from the site of the intervention [7].
The FIRE (Fire Information and Rescue Equipment) system takes advantage of using the SmokeNet sensor network. These are sensors provided in the building within the framework of anti-fire prevention. The sensors must be installed in every room and separated one from another by about $10 \mathrm{~m}$. The FIRE system is able to use the network of these sensors for the locating of the firefighters in the building. The system is simultaneously able to acquire further data from the Smoke Network as, e.g., information of what rooms have been hit by the fire. The system also includes a miniature display FireEye which the firefighter is equipped with and thus, is able to clearly monitor important data. The system is currently under development and testing [8].

ProeTex is a project which is implemented under the support of the $6^{\text {th }}$ framework programme of the EU. The project is primarily focused on the development of "smart textiles" which could be employed in the future for the production of protective clothes and auxiliaries for firefighting teams. Textile sensors developed within the framework of this project are particularly aimed at scanning basic life functions, physiological parameters and potential activity of chemical hazards (toxic substances, etc.) along with problems of power supply units for their equipment [9]. In accordance with information available, some the textile sensors are interconnected in a classical way through cables, which facilitates the design and implementation of the system to a certain extent (the complicated stage of designing and testing the radiofrequency interface is eliminated; this solution has further positive effects on the electric power consumption, since compared to the wireless transfer, its energy efficacy is principally higher). However, a disadvantage of this solution is a certain restriction to the user and significant increase in effects on his comfort. Cables serving the interconnection of particular points are relatively susceptible to the damage (for example, material damage due to fatigue of the conductors themselves by mechanical stress in the same parts of clothing).

\section{Surveillance system for supporting intervention by and training of Integrated Rescue System - FlexiGuard}

FlexiGuard is an abbreviated name of the project which is currently being designed by a team of investigators at the Faculty of Biomedical Engineering, Czech Technical University in Prague. The target of the project is the development of a telemetric monitoring system in a more resistant form, allowing for the locating of particular members of the rescue team, monitoring of their health-physiological parameters (pulse, pressure, skin resistance - sweating, temperature), automatic detection and signalling of hazard conditions, such as physical exhaustion, excess stress, overheating, etc., in real time and under extreme circumstances. Further, it will make the differentiation of the nature and intensity of their motion (lying, standing, running, 
crawling, etc.) possible, including the identification of topical and total energy output, monitoring of environmental parameters (temperature, smoke, etc.) and further conditions depending on actual requirements of selected teams of the Integrated Rescue System.

Expected users of the resulting project were defined in the course of the project preparation. The possibilities of its use were particularly based on the needs of training particular types of users and methods developed for use in possible extraordinary events and crisis situations both common and uncommon. The expected users of the project results with specifying the framework of the use are outlined below.

- Health rescue service - training, interventions of members of the health rescue service and monitoring of people injured in mass accidents, etc.

- Firefighting emergency teams - training, protection in all the types of interventions, particularly interventions with the use of respirators, physically tedious interventions, extensive fires, floods, mass accidents.

- Mountain rescue service - training, navigation and location, prevention of collapse and physical exhaustion under conditions of high altitudes above the sea level.

- Police corps - training, intervention of special teams, largeextent interventions.

- Mining rescue service - training, interventions under the ground, possibilities of location in smoky environment.

- Water rescue service - training, work under the water surface.

- Army - training and special interventions.

Principles of the function and continuous results of the FlexiGuard surveillance system

The surveillance system is focused on establishing a sensor network allowing for the wireless transfer of physiological and environmental quantities from the user body or from a close vicinity (for example, from clothes). The wireless solution was chosen with respect to the possibility of system integration into equipment and resistance to the mechanical damage.

Particular quantities, i.e., technical, physiological, environmental, etc., are scanned with the help of a network of sensors and data from sensors measured in this way and are digitalized and transferred wirelessly by a modular scanning unit: for details see Fig. 1.

The topology of the system testing sample is based on the BAN (Body Area Network) and the data is thus scanned from probands with the help of a network of sensors (in the future incorporated for example in the gear of the Integrated Rescue System member), and signals from the sensors can be digitalized and transferred within the framework of the BAN network node to the modular scanning unit wirelessly, by a short-range communication interface.
Every member of the team has his own modular scanning unit to which data from appropriate autonomous sensors are transferred. The data is transferred from the modular scanning unit to the local visualizing unit and processed by the software to simple output data, useful, for example, for the intervention commander. A possibility of the transfer to a distanced visualizing unit is also expected, for example, to the controlling position, if possible.

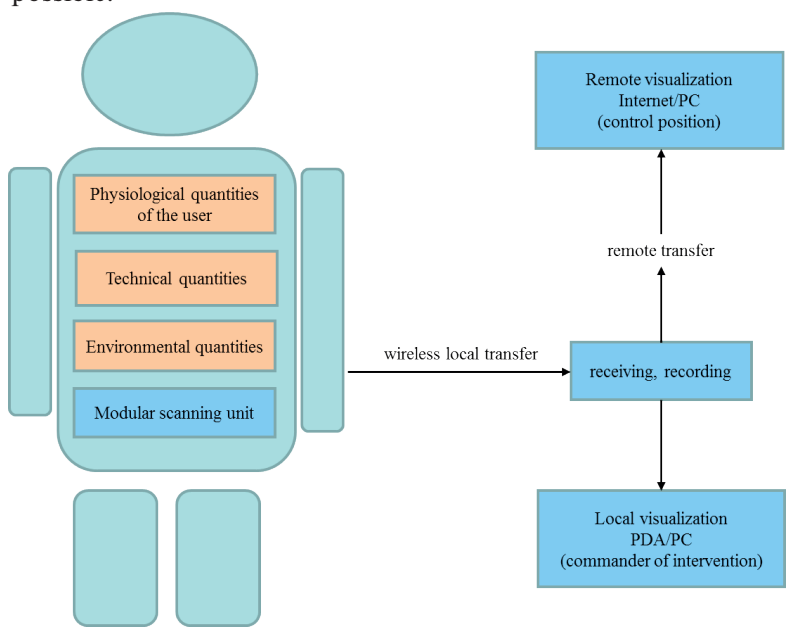

Fig. 1 Principles of the FlexiGuard surveillance system function

The data can be distributed in two independent manners. The first one dispatches in real time to the visualizing unit with the help of a long-range wireless network (on-line regimen). There is also a possibility to store the data on a recording medium (microSD) which is a part of the modular scanning unit and to read them later with the help of the USB connection to the PC (off-line regimen).

Within the framework of the project, several variants of visualization (analysing) units were prepared and verified in the form of a PC, more resisting notebook and PDA which were equipped with a specially designed and debugged application software. For purposes of testing the basic modular scanning unit, at the present stage of the project, the notebook with enhanced resistance was most successful as the visualization unit. The visualization unit in its basic variant is able to receive, display and file data from connected modular scanning units in real time.

The visualizing unit software itself is arranged as a component system and makes possible the subsequent extension by advanced reconstructing and visualizing algorithms which are able to display data received in real time, to file them on a memory medium and to evaluate them in real time in accordance with the requirements of future application variants of the system. The software for the visualizing unit is also ready to work with a remote data base (administration of personal safety profiles of users and data). In the basic variant of the supporting software visualizing unit, at this stage of the project solution, processing and analysis of the following quantities were implemented: 
- topical load - calculation based on the Astrand-Ryhming chart; input parameters such as the mass, height, age, sex, maximum pulse frequency, maximum oxygen consumption (VO2max), energy equivalent for oxygen and topical pulse frequency,

- integral load - aggregation of the topical load in defined sliding time window,

- body position - differentiation between several positions (lying, standing, etc.),

- load - as calculated from accelerometers data,

- actual condition of the battery and estimate of its endurance,

- body temperature - measured by a thermistor in the zone of the thorax,

- external temperature (temperature of the environment),

- adaptive processing of the pulse frequency, elimination of artefacts.

The principle of the interconnection of modular scanning units with the visualizing unit is shown in Fig. 2. The modular scanning unit is equipped with several interfaces (wireless BAN network, A/D converters) for connecting various sensor types. Any sensor equipped with an appropriate interface can be theoretically connected. Every member of the team has his own modular scanning unit to which data is sent from relevant nodes of the BAN network, i.e., autonomous sensors. There is also a possibility of directly connecting the analogue sensors without using a further node of the BAN network. The modular scanning unit is also equipped with a wireless communication interface for the communication with the visualizing unit.

The connection of sensors (BAN nodes) to the modular scanning unit is completely automatized. After switching on the sensor and situation within the wireless range of the unit, the sensors are automatically connected to the unit. In the course of the measurement, particular sensors (BAN nodes) can be arbitrarily connected or disconnected. The disconnection of sensors is performed only by their deactivation or removal beyond the range of the radio communication. The re-connection is carried out by switching on or returning into the range of the modular scanning unit without necessary re-starting or affecting already connected sensors.

Within the framework of the project, the basic hardware platform was provided for the research and development of particular required measurements and communication modules with detection algorithms of subsequent debugging application variants. The testing sample arranged, already in the basic variant, makes it possible for the on-line monitoring of basic physiological and environmental parameters:

- ECG and pulse frequency derived from it - a source signal for monitoring the adaptability of the Integrated Rescue System members to load and stress situation and for automatized estimate of the energy output,
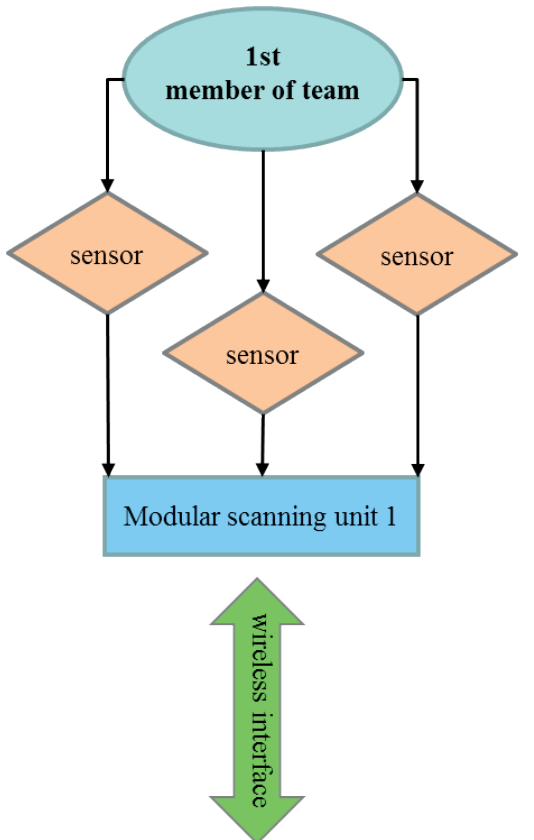
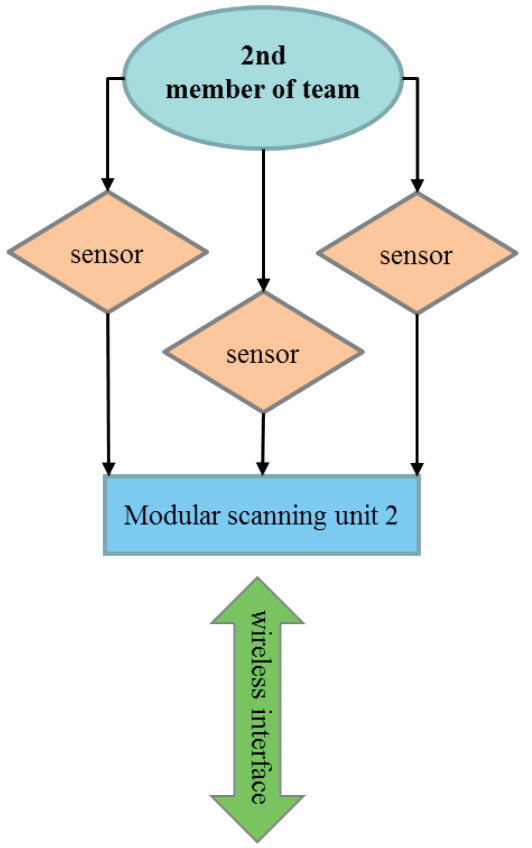

Visualization unit

Laptop, PDA, Tablet, SmartPhone
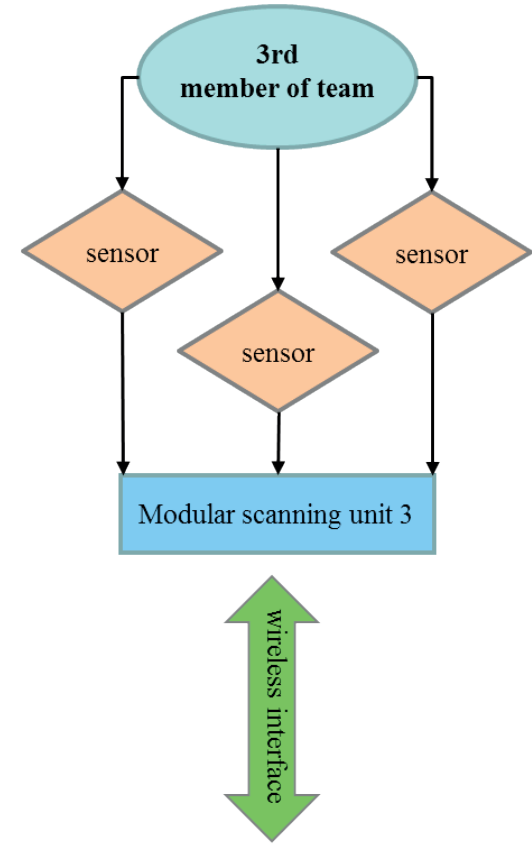

Fig. 2 Modular concept of the FlexiGuard surveillance system 
- actigram measured by a tri-axial accelerometer, serving as a source signal for subsequent derivation of information about the nature and intensity of the physical activity (rest, walking, running, crawling), about the body position (lying, standing) and about the physical load intensity,

- body temperature, temperature of the environment and air humidity (can be measured at several points - a source signal for the assessment of the thermal comfort or of overheating selected parts, for example, the gear, etc.).

Modules have also been prepared for the measurement of the blood saturation with oxygen, surface scanning of the myogram, blood pressure, breathing frequency, skin resistance, etc. The set of these basic parameters is quite open, and based on continuously providing feedback in debugging of the system with members of the Integrated Rescue System, the set of source signals can be supplemented and refined on demand. For example, from the present analysis of the needs of the Fire Rescue Service of the Czech Republic it follows that for the application variant, the training monitor can be suitably supplemented, for example, by scanning the position of the proband (firefighter) or detecting selected gases. There is an important possibility to use the system for monitoring exposure to carbon monoxide through the course of the intervention without using an autonomous breathing apparatus. In accordance with the literature, there is a risk that particularly in long-term and repeated exposures, its effects are frequently underestimated due to its physicochemical characteristics (carbonmonoxide is odourless, colourless and non-irritating).

\section{Technical verification of the FlexiGuard surveillance system function}

Within the scope of the project, in addition to the proposal of the implementation of outputs from the visualizing unit, the function of the modular scanning unit was technically verified, which serves for scanning data from individual sensors and for their transfer just into the visualization unit. The technical verification of the function was provided with the help of laboratory and semi-field experiments. The team of investigators performed a number of controlled technical experiments simulating different loading situations in the laboratory (rest condition, running, knee bends, crawling, etc.). Data from these experiments will further serve in checking the algorithms of processing and also in the consideration of the use of the quantity monitored by measurements in practice.

Within the framework of the first field experiments, technical parameters of the basic scanning unit including the capacity of the communication channel were verified in the pilot plant environment. Information was acquired concerning the suitable situation of sensors, the physical arrangement and ergonomic requirements for the measuring equipment. The algorithm for the estimation of the load or energy output was also tested. The results and experience acquired will be immediately employed at the next stages of the project solution, on the one hand for the optimization of the testing sample, and also in the development of end application variants of the system (training and intervention monitor).

\section{Conclusion}

The surveillance system developed is designed in a modular form with the possibility of easy extension by further application hardware and software modules based on the requirements of particular end users. It is thus possible to add, for example, a sensor for the detection of selected groups of dangerous substances, or to arrange the software for outputs from data measured and thus to establish a system "tailored to" a particular user.

In cooperation with selected components of the Integrated Rescue System, application requirements will be further developed for the training or intervention module in order that the resulting application variants of the system might be used in practice while enhancing the safety of the intervening members of the Integrated Rescue System teams.

The resulting training module will be applicable to individual monitoring and quantification of the course of training particular members of the team in real time, to determining the immediate reaction to different situations (stress, load), to recording the course of the training and subsequent long-term monitoring of the course of particular parameters during the training process - determination of the progress of particular members in the course of training, evaluation of the training efficacy, and the determination of individual limit parameters of particular team members at a certain stage of the training.

The resulting intervention module will be applicable as a supporting, protecting and surveillance tool for members of selected teams of the Integrated Rescue System through the course of the intervention. The system operation will be based on the principle of monitoring physiological parameters in individual intervening persons and supplementary information on the surrounding environment. It will also be able to provide automatized detection of critical conditions (overheating, physical exhaustion, extreme stress) their automatic signalization and location of particular members of the team.

The whole surveillance system is designed on the one hand for supporting the decision process of the commander of the Integrated Rescue System intervention in real time and also for back evaluation of the course of the intervention and for acquiring and visualizing summarized and individual data on the behaviour of members of the team in managing different situations through the course of the intervention and in training.

\section{Acknowledgements}

This work was supported by Project No. VG20102015002, Ministry of the Interior of the Czech Republic. 


\section{References}

[1] RISTVEJ, J., ZAGORECKI, A.: Information Systems for Crisis Management - Current Applications and Future Directions. Communications - Scientific Letters of the University of Zilina, vol. 13, No. 2, pp. 59-63, 2011, ISSN 1335-4205.

[2] HON, Z. et al.: Biotelemetry and its Use for Rescue Teams. Urgent Medicine, vol. 16, No 1, 2013, pp. 29-32, ISSN $1212-1924$.

[3] REHAK, D., DUDACEK, A., POLEDNAK, P.: A Multipurpose Robotic Vehicle for the Rescue of Persons and Interventions in Emergency Situations. Communications - Scientific Letters of the University of Zilina, vol. 15, No. 1, pp. 103-109, 2013, ISSN 13354205.

[4] KLANN, M. et al.: LifeNet: An Ad-hoc Sensor Network and Wearable System to Provide Firefighters with Navigation Support. Adjunct Proc. Ubicomp Innsbruck, Austria. 2007. pp. 124-127. Available at: http://eprints.lancs.ac.uk/13037/2/2007-LifeNet.pdf.

[5] KLANN, M.: Tactical Navigation Support for Firefighters: The LifeNet Ad-Hoc Sensor-Network and Wearable System. J. Loffler and M. Klann. Mobile Response. Berlin: Springer, 2009. pp. 41-56, ISBN 978-3-642-00439-1.

[6] TIA, G. et al.: Wireless Medical Sensor Networks in Emergency Response: Implementation and Pilot Results. Proc. 2008 IEEE Intern. Conference on Technologies for Homeland Security. May 2008, pp. 187-192. Available at: http://www.cs.jhu.edu/ $1 j h /$ paper/ ieeehst2008.pdf.

[7] KEWEI, S., WEISONG, S., WATKINS. O.: Using Wireless Sensor Networks for Fire Rescue Applications: Requirements and Challenges. Electro/information Technology, 2006 IEEE Intern. Conference, May 2006, pp. 239-244. Available at: http://ocu-stars. okcu.edu/ksha/sha06-firenet.pdf.

[8] WILSON, J., et al.: A Wireless Sensor Network and Incident Command Interface for Urban Firefighting. 2007 Fourth Annual Intern. Conference on mobile And Ubiquitous Systems: Networking \& Services, 2007, pp. 19-25. ISBN 978-1-4244-1024-8. Available at: http://fire.me.berkeley.edu/Misc/Mobiquitous2007-JWilson.pdf.

[9] Advanced e-Textiles for Firefighters and Civilian Victims [online], [vid. 09. 09. 2014]. Available at: http://proetex.org. 